\title{
Community-Based Referential Music Making with Limited-Resource Adolescents: A Pilot Study
}

\author{
Natasha Thomas, PHD, MT-BC
}

\begin{abstract}
Black/African American adolescents from limited-resource communities face challenges and circumstances that are unique to their racialization and socioeconomic status; this merits communityengaged resources, such as community music therapy, that are equally unique in creating culturally responsive opportunities for limited-resource adolescents to engage socially with peers and experience meaningful success in a safe, supportive environment. The purpose of this study was to pilot and explore the feasibility of and behavioral processes in a community-based referential music-making intervention for limited-resource adolescents labeled as "at-risk." The methods consisted of a concurrent nested (embedded) mixed methods design based on the principles of participatory actions research (PAR), during which qualitative data were collected during 8 focus group style music-making sessions. Quantitative data assessing self-efficacy were collected prior to first and following the 8th music-making session. The validity of quantitative results was challenged by the lowered reading level of participants and a high amount of mis-labeled (and thus unusable) data. Qualitative data suggest 3 themes, including creating community, artistic prioritization, and pride. All results were impacted by issues, such as inconsistent attendance and malfunctioning recording equipment. Nevertheless, participants expressed a collective desire to share their work with their community group. Discussion points are raised including how participants in this community music therapy-based approach were able to create and direct their own stories. The implementation of community music therapy approaches seems a valuable way to bring authentic representations of limited-resource adolescent participants into clinical practice.
\end{abstract}

Keywords: adolescents, African Americans, music therapy, resource communities, participatory action research

\section{Introduction}

Trauma, in the form of systemic oppression, has a uniquely heavy impact on racially and socioeconomically marginalized adolescents (Darensbourg \& Blake, 2013; Elsaesser et al., 2016). Adolescents who live in predominantly Black or African American communities and characterized as being "at-risk" are often exposed to this trauma in ways that are unique to their racialization and socioeconomic status, including systemic racism, poverty, and exposure to violence, which are interrelated (Burch, 2014; Elsaesser et al., 2016). This trauma can produce a domino effect where behaviors such as truancy and substance abuse are compounded by environmental and systemic issues, impacting academic performance, self-efficacy, and general quality of life (Aramovich, 2014; Harrist \&

This is the author's manuscript of the work published in final edited form as:

Thomas, N. (2020). Community-Based Referential Music Making with Limited-Resource Adolescents: A Pilot Study. Music Therapy Perspectives. https://doi.org/10.1093/mtp/miaa016 
Bradley, 2002; Ladson-Billings, 2006; Steele, 2010; Vaillancourt, Brittain, McDougall, \& Duku, 2013). This cascade of behaviors and issues forms the basis of the term "at-risk," which is widely used by clinicians to refer to these youth and their needs.

However, researchers caution against speculations (Ladson-Billings, 2006; Elsaesser et al., 2016; Steele, 2010) or oversimplifications of backgrounds or behavioral traits that children might exhibit, in favor of prioritizing the voices of these children themselves. Adolescents from limited-resource communities struggle to define and empower themselves in environments that they believe do not value or support them. Reframing the language used to speak about them as individuals - that is, utilizing the term "limited-resource adolescents" as opposed to "at-risk youth" is one way to center the focus on addressing youth needs for the valuing and support of their voices (Thomas, 2019).

Music is just one form of voice for Black American culture and identity (Dixon et al., 2009; Donnetrice, 2012), woven into the fabric of Black America, from coded communication during slavery to overt messaging of emancipation, community empowerment, identity, and resistance against oppression (Pyatak \& Muccitelli, 2011; Towns, 2015). Black musical practices, such as those found in Hip Hop Culture ${ }^{1}$, build on the Civil Rights and Black Power movements (Rabaka, 2013) and have become largely mainstream. However, the coded language within them remains in a fluid flux between marginalization to popular consumption and may or may not be recognized by individuals from outside the Black community.

This divide between the popularity of Black musical culture in the mainstream and the marginalization of those who birth it manifests in a variety of ways, including the underrepresentation of Black American adolescents in school-based music-making opportunities. It is possible that youth may opt out of such experiences (Saunders, 2010), or they may be excluded entirely from them (Gaztambide-Fernández, Saifer, \& Desai, 2013; Lorah, Sanders, \& Morrison, 2014). Regardless, Black American adolescents assert their freedom to choose what they listen to, how they listen to it, and who they share those experiences with, on their own time and terms, as research indicates adolescents generally do (McFerran, Garrido, O'Grady, Grocke, \& Sawyer, 2015). The impact of music's reinforcing or transformative effect can be reflected in the higher sense of collective self-esteem demonstrated by Black or African American adolescents who describe themselves as being avid consumers of Hip Hop-music is a major part of how they develop and assert their own sense of self and related beliefs, which can have a major impact on their overall quality of life (Dixon et al., 2009). Yet, it seems apparent that lack of opportunity, cultural relevance, and access sustains an overrepresentation of white, upper-middle-class students in schoolbased music ensembles, as well as the research about them (Elpus \& Abril, 2011; lerardi, Bottos, \& O’Brian, 2007).

Community music therapy, as first described by Ansdell (2002), regards the individual and community as part of a holistic continuum of health, which holds some promise in breaking through this white-favoring overrepresentation. Through taking part in any capacity of music making, whether performance, listening, or other forms of engagement under the umbrella of "musicking" as defined by Christopher Small (1998), participants can be empowered as primary stakeholders in co-constructing performative works. These works can then be grounded in activist aspirational ethics and reflexive in nature, locating, centering, and engaging directly with the people and resources of the community in ways that are affirming and sustaining of cultural needs, priorities, and values (Kenny, 2004).

\section{Positioning Statement and Research Questions}


Given the lack of opportunity and access to school-based music-making experiences for Black/African American limited-resource adolescents, and a gap in the literature regarding the specific experiences of this uniquely racialized and socioeconomically situated group, this study sought to explore the feasibility of a community-based referential music-making intervention focused on the self-concept (self-efficacy specifically, as a system of belief about the self), and influenced by elements of participatory action research, which involves the reflexive challenging of traditional power differentials in the design, implementation, interpretation, and dissemination of research (McTaggart, 1997). Stige (2002) advocates for participatory action research as being particularly relevant to culturally centered music therapy practices. This occurs through the promotion of democratic decision making and more proactive attempts on the part of researchers to engage their participants more actively in every aspect of the research project.

The primary researcher for this project was a Black, cisgendered, queer woman, and board-certified music therapist (MT-BC) with a PhD in Expressive Therapies, living and working at the time in New Orleans, LA, where the study took place. The research questions were as follows:

1. What is the feasibility of utilizing a Likert-scale-based tool for measuring self-concepts (such as self-efficacy) as a foundation for goal setting in music therapy with Black/African American adolescents from limited-resource communities?

2. What processes might emerge in the formation of a community-based referential music-making intervention that prioritizes the voices and priorities of Black/African American adolescent participants?

\section{Methods}

\section{Participants}

Participants were purposefully recruited via convenience sampling (Hoskins \& Mariano, 2004) of students 11 to 14 years of age by staff at a community agency for youth labeled "at-risk" in New Orleans, LA. Participant recruitment involved a verbal invitation from staff for a "music therapy research group" to be scheduled to meet right before dinner time on Tuesday evenings for 30 to 45 minutes. There were no other inclusion criteria or exclusion. This initially resulted in a group of 10 participants, though the final group included only 8 participants ( 3 identifying as male and 5 female) ranging in age from 11 to 13 years old. All participants identified themselves as Black or African American. All participants had engaged in music therapy sessions facilitated by the primary researcher's music therapy students from their institution in the semester prior to the research group and were familiar with the primary researcher's presence at the facility, even though she had not previously facilitated any full previous sessions with them.

The Institutional Review Board of Loyola University New Orleans approved the study. Study information sheets, general consent forms, and photo/recording release forms were submitted to and signed by the legal guardians. After an initial informational meeting, participants also signed assent forms and were asked at the start of every subsequent session to affirm verbally that they assented to their participation in that portion of the study for the day.

\section{Setting}


The setting for this study was an after-school community program provided by an organization based in New Orleans, LA, which will be referred to in this paper as the "Creative Community Project," or CCP. Programming at the CCP is led by a variety of paid and volunteer staff, with two or more leaders assigned groups of up to 10 student participants, divided by age. Participants typically rotated between spaces and classes, such as homework support, life skills, and arts-based or other recreational classes. The facilitator for the community music therapy sessions, the primary researcher, was a board-certified music therapist (MT-BC) with a PhD in Expressive Therapies, who also identified as Black.

\section{Instrument: The New General Self Efficacy Scale (NGSE)}

In choosing a quantitative measure for this study, the primary researcher chose to focus her attention on the construct of self-efficacy, which is an individual's "estimate of (their) overall ability to perform successfully in a wide variety of achievement situations" (Chen, et al., 2004, p. 375). Self-efficacy is distinguishable from other constructs such as self-esteem or locus of control and other outcome-based self-expectancies, as those are more focused on self-worth, or self-control over various external contingencies, rather than belief in one's capability. The belief-based nature of self-efficacy was of particular interest to the researcher, given how Black youth from limited-resource communities are often excluded from (or may opt out of) school-based music-making opportunities, which may be informed by beliefs (whether theirs or others') about their capability to succeed in such spaces.

The tool chosen to measure participants' sense of self-efficacy was the New General Self Efficacy Scale (NGSE). The NGSE was created and normed for adults and adolescents at a seventh-grade reading level, containing eight items relating to generalized self-efficacy. These two qualifying elements (reading level and the number of items) made the NGSE more appealing to the researcher than other measures that contained more elements and were normed for higher reading levels. The eight items of the NGSE were as follows: the ability to achieve goals, accomplishing difficult tasks, obtaining outcomes of personal importance, succeeding at endeavors, successfully overcoming challenges, confidence in the ability to perform effectively on multiple tasks, doing well on tasks when compared with other people, and performing well despite challenges. Each item on the measure is presented with a 5-point Likert scale of responses ranging from I have no confidence in my ability to do this $(0)$ to I am very confident in my ability to do this (5).

\section{Plan}

Participants met for a total of eight sessions. Specific session formats fluctuated week to week as the project progressed and are presented in Supplementary Table 1.1, available online.

In general, all sessions involved the establishment of goals for the day at the start, always based on participant suggestions, with as minimal guidance from the facilitator as possible. This was in keeping with participatory actions research (PAR) methodology, and the project being participatory, the first letter of Stige and Aaro's (2012) "PREPARE" model for community music therapy. The first session involved completing the NGSE, followed by sessions focusing on discussing results and establishing the musical preferences and general priorities of the group based on NGSE results. Later sessions focused on the conceptualization and execution of a plan for a participant-directed video featuring original music and dance performance elements. 
Participants took responsibility for determining the direction of the project and assigning roles for themselves, including beat making and music production, songwriting, and rapping or dancing for the video, as well as post-production and editing of the final video product. One participant was typically designated as a note-keeper for each session, in addition to the primary researcher. Some examples of note-taking templates used by participants in this study are provided in the Supplementary Appendices of this paper, where the lyrics for the resulting piece of music can also be found. During the final session, participants re-took the NGSE, analyzed and discussed the results collaboratively with the primary researcher, and discussed the sharing and finalizing of the group project, including the possibility of presenting the video to their community at an end of year parent event. This was in keeping with the resource-oriented, ecological, and performative elements of community music therapy as described in the "PREPARE" acronym from Stige and Aaro (2012).

\section{Data}

Analysis of the NGSE was limited due to some confusion on the part of participants that resulted in inconsistent identification between their pre and post-tests. Thus, the majority of their submissions were wholly anonymous and could not be compared. Therefore, only descriptive statistics (means and standard deviations) were analyzed.

The primary researcher viewed and transcribed the session videos, striving to capture all audio and visual session content verbatim. The researcher additionally endeavored to note the direction of speech whenever it was not made obvious within the dialog. Dialect proved to be a challenge with some passages of video, being that the researcher was not originally from the region. So, while a denaturalized transcription that included unique pronunciations and pauses in the speech was the goal, in some instances, a parenthetical including a naturalized "translation" was provided after any passages, which may have been difficult for anyone unfamiliar with the local vernacular to understand.

Analysis of videos and transcripts was conducted via a manual open coding process, reading, and rereading through the transcripts several times, underlining and circling words, ideas, and actions seen repeated multiple times throughout the document. Themes were then organized under an axial coding model, as described by Creswell (2007) to determine the causal elements of each theme's mention or occurrence, how it was responded to, any additional influences on these responses, and related consequences. From that point, thematic elements with the highest number of recurrences were highlighted as central phenomena, then written up in a report-style format that could be returned to the group for their discussion and validation at the next session, in keeping with the consistent repetitive cycle of planning, acting, observing, and evaluating in PAR as described by McTaggart (1997). This also paralleled the activist, reflective, and ethics-driven qualities that make up the final letters of Stige and Aaro's "PREPARE" acronym for community music therapy.

\section{Results}

\section{Feasibility of the NGSE}

\section{Reading level.}

The NGSE was normed for use with adults and adolescents at a seventh-grade reading level. While the majority of participants in the project were seventh graders, it was discovered early in the process that they were not comfortable with that level of reading material. Thus, the researcher read all questions 
aloud and sought to define any terms at which participants expressed confusion in more simplified terms.

\section{Data collection.}

Supplementary Table 1.2, available online, illustrates the pre and post-test results for the NGSE taken on sessions one and eight of the project.

Eight participants took the NGSE on session one, but only five were present to do so on session eight. Additionally, in the post-test NGSE, only two participants wrote their names, and three did not. This lack of consistency between the pre and post tests makes it impossible to reliably compare the results, though it is interesting to note that all NGSE areas, with the exception of one, saw an increase in the average number of participants who expressed confidence in their ability to perform the identified skill. Interestingly, the one area that did not see this increase was the skill area chosen by the group as a goal to address musically (overcoming challenges). Given the issues with participants not consistently labeling their pre and post-test submission forms, however, results could not be verified. Ultimately, considering the challenges that the tool's reading comprehension caused for the group, the validity of these results is also questionable.

\section{Additional feasibility issues.}

Due to a desire to first get to know participants without the pressure of being recorded, Session one was not video-recorded and technical difficulties rendered video footage from Session Three un-viewable. Session six was fully recorded, but participants were not entirely viewable within the video frame. Additionally, attendance at all sessions was inconsistent, due to a variety of transportation and scheduling issues; some days there would only be one or two students in attendance, other days all eight students would be present, with additional students from other groups occasionally "dropping by" to hear what was being worked on. One participant self-selected to withdraw from the project after the first few sessions, and another was removed from the community agency as a whole for behavioral reasons unrelated to the study. Due to staffing challenges, co-leadership was also inconsistent. Sometimes a community volunteer was present; other sessions were facilitated solely by the primary researcher.

\section{Process Themes (Finding Our Collective Voice)}

Central themes emerging across sessions included creating community (coming together), artistic prioritization, and the desire to be affirm and be affirmed (witnessing).

\section{Creating community (coming together).}

While participants largely knew each other from general classes and events at the CCP, this was their first time working together in this capacity, and in the first few weeks of the study, the ways in which participants interacted with each other were primarily physical and/or nonverbal in nature, as they came to know each other more deeply and establish roles in this new context. These behaviors could appear positive, negative, or neutral. For instance, positive behaviors might include smiles or laughter, whereas negative behaviors might present as frustrated affects and sounds, and neutral interactions would present as limited responses in either direction. Behaviors could further be clarified within these three categories by whether the interaction was productive, disruptive, or escalating, as experiences 
that might have seemed initially disruptive could turn out to be positive or productive, and initially neutral interactions could escalate into negative ones. This resulted in five final subcategories of nonverbal behavior: (1) fully positive and productive throughout, (2) fully positive/productive but initially disruptive, (3) initially neutral, escalating to negative, (4) fully negative but non-disruptive, and (5) fully negative and disruptive. These weighted terms were considered carefully and revealed important details about how participants interacted with each other, which shaped how the researcher might encourage interaction in future sessions.

For example, at one point in the second session of the project, participants spontaneously engaged in a game of "Rock, Paper, Scissors" to help determine who was going to take notes for the session, a response that was not requested and which disrupted the process of handing out note-taking sheets to all the students, as the researcher had planned to do. However, seeing how the group chose to instead "elect" a single note-taker for the group, via this "tournament" of sorts, it became a tool to possibly be of use for later decisions the group might need to make.

As the sessions progressed, and participants were given the space and time to get to know each other and explore alternatives to physical action, participants seemed to develop an increasing amount of verbal strategies to assert what they wanted and appreciated (and vice-versa) in the safe space that sessions provided. These strategies were demonstrated in writing and out loud, through general participant engagement after prompts from the primary researcher or another peer, or participant volunteered information or engagement, without prompting. The written engagement was the first method by which participants were encouraged by the primary researcher to voice individual perspectives and work out disagreements, including responses to such prompts as defining items relating to the project (such as "self-efficacy" and "referential music making"). Participants were additionally prompted to write out their choices for what items in the NGSE they regarded as priorities (resulting in a unanimous vote to reference overcoming challenges in the project). When there was confusion or disagreement, the primary researcher would encourage the group with labels or definitions for the verbiage or behavior they were observing, providing examples and/or follow-up questions relating to tangible ways in which the ideas or needs being expressed could continue to be productively explored (either verbally or with musical choices, etc). Once elaborated on in this way, participants seemed more confident in offering increasingly nuanced and deeply connected questions and contributions to the artistic priorities of the project as it evolved.

Participants responded both verbally and in writing to prompts for what kind of musical product the group wanted to create (a music video, also unanimously approved by the group), and what sorts of artists, songs, or styles the group considered influences on the type of project they wanted to create, for which participants-all of whom identified as Black/African American-chose from the Rap or Hip Hop genres. Written responses to prompts peaked at the fourth session, where participants were noted to abandon writing as a primary means of negotiation and instead seek to prioritize verbally asserting their needs and roles on the audio and visual aspects of the project, which began in earnest around that time. Verbal engagement from that point forward was largely participant initiated and not prompted, including validation or disagreement between peers or between participants and the primary researcher, or suggestions for lyrics or other content for the project. This prioritization of verbal engagement seemed representative of the trust and sense of community that participants were building with and between each other, and increased until the final session. 


\section{Artistic prioritization.}

The music making in this project seemed to consistently serve as a clarifier of confusion and motivator for participants' increasing verbal engagement, and thus also emerged as a stand-alone central theme, reflecting how individual participants viewed themselves and their relationships to their peers (and the project as a whole). From the suggestions made for songs or artists who might relate to the chosen goal of overcoming challenges (such as Kendrick Lamar or Da Boogie wit da Hoodie), to the roles individual participants chose to take in their own original songwriting process, and even how they conducted themselves in the processes inherent in these roles, they were telling each other who they were and how they viewed the world.

As an example, Dee Dee (a pseudonym chosen by the participant) volunteered to devise lyrics for the project, and did so at home between sessions, coming to session five with a rap she had written at home after the priority focus of overcoming challenges and a beat for the song had been voted on by the group in the project's fourth session. This could have been done in session, but such was how seriously Dee Dee took her role. The group seemed to honor this, cheering her on when she demonstrated the rap to the group. In particular, another participant, Red, was noted to start rhythmically hollering "Yeah! Yeah!" as Dee Dee performed, which ultimately became the hook of the song, with the lyrics "Yeah, yeah, now we're overcoming."

In general, participants seemed to take great pride in putting the project together, demonstrating meticulous attention to detail at every stage. The final product, which was a Hip Hop style music video titled simply "Overcoming," featured original rapping and choreography that was both written and produced entirely by them. The full lyrics of the song can be found in the Supplementary Appendices.

\section{The desire to affirm and be affirmed (witnessing).}

Participants were pleased with the final product of the music video, with one participant being noted to say saying "Good. It's good," during the last session, and various participants could be observed nodding their heads when asked what they thought of the final version of the music video when shown to them by the primary researcher. These were youth who had begun this experience largely nonverbally, with shrugs and ambivalence their common currency, so any verbal expression of pleasure at something was notable. Some participants were even eager to have their families and peers view the final music video, with three youth who were present at the last session suggesting that the video be shared publicly by posting it to the CCP's Youtube page so that it could be viewed by the community at large. Some participants were also keen to have their full names used in the video's credits and expressed frustration when told that the confidentiality agreements that had been signed between their parents and the researcher would prohibit this. The decision to use "stage names" for the project was thus chosen as a compromise to this, allowing the students to be able to identify themselves in the project as they saw fit, without technically breaking with the researcher's ethical responsibilities. It seemed important to participants that they not only be able to have their work witnessed, but also to be able to stand by it with their physical bodies and names, and receive credit for that work.

\section{Discussion}

In general, the artistic process of creating a piece of Hip Hop music that referenced participants lived experiences enabled them to give an authentic voice to their identities and experiences as Black/African 
Americans with ties to Hip Hop culture (Dixon et al., 2009; Donnetrice, 2012; Rabaka, 2013). This process mirrored the healthy development of self-concept found in previous research, through the creating of new, community-based foundations for evolving and collaborative ideas and expressions (Ansdell, 2002; Hense, 2015; lerardi et al., 2007). Participants, by and large, were increasingly engaged across a majority of elements of the project, and democratically asserted their desires across divides, in ways that created a more mutually beneficial experience for the whole, which is a core goal of participatory research (McTaggart, 1997). Additionally, the performative and culturally relevant premise of the project encouraged youth to connect with each other and their community in new ways, with their active participation increasing their access to-and use of-interpersonal and intracommunity resources in reflective and sustaining ways. These results were very much in keeping with the tenants of community music therapy and culturally relevant pedagogy (Ladson-Billings, 2006; Stige \& Aaro, 2012). This type of collaborative process yielded a sense of pride and empowerment such that youth wanted not only for their community to witness it, but also to affirm it for themselves. This kind of experience of selfaffirmed "success" is one of the core building blocks to a healthy sense of self-efficacy and has been found to lead to positive strides academically and in other areas with which children and adolescents from limited-resource communities frequently struggle (Bandura, 2005; Vaillancourt et al., 2013).

\section{Challenges}

Most notable among this project's limitations was the invalidity of the NGSE. The published reading level of the instrument did not match the participant's reading capacity. Thus, future studies need to consider either an alternate standardized measure or a researcher-created instrument, which carries its own issues. The lack of a suitable instrument points to a general lack of self-efficacy research with this population and their unique challenges in general.

The attendance of participants was not consistent throughout the study period, which did not seem to be outside of natural attrition for research in general. This could possibly have been addressed, however, by either having multiple session times or opportunities per week for participants to engage or extending the overall length of the project, so that participants could more intently build a single weekly time into their schedules. The size of the group at its largest also made for significant challenges in behavior management and transcription of session materials. A smaller group of perhaps 5 to 6 participants may have been more feasible.

\section{Clinical Implications and Conclusions}

Adolescents who have been labeled as "at-risk" of academic failure or general health risks due to poverty or trauma display a "domino effect" of challenges from disruptions created by the systemic nature of their racialization and socioeconomic status (Aramovich, 2014; Ladson-Billings, 2006; Steele, 2010; Vaillancourt et al., 2013). Music has historically served as a unique and valuable outlet for Black/African Americans (Dixon et al., 2009; Donnetrice, 2012; Pyatak \& Muccitelli, 2011; Rabaka, 2013; Towns, 2015) and for adolescents to explore and define their own feelings and needs (McFerran et al., 2015). Participatory-based projects like those found in community music therapy (Ansdell, 2002) that regard the individual and community as part of a holistic continuum of health additionally demonstrate the value of prioritizing those voices at the center of the research process, toward the future of clinical work that invites participants to be directors of their own stories. 
Clinicians working with Black/African American limited-resource youth may wish to consider the tenants of Participatory Action Research, in conjunction with community music therapy in how they approach initiating and engaging with these youth to address their needs. Particular actions that may be of value could be to interrogate the process of session planning and consider how participants might be more engaged in that process. Perhaps through the use of tools such as the NGSE or other self-concept measurements, participants could be empowered to establish their own goals and designate note-takers of their own to assist in documenting their process toward those outcomes. Then, as logistical processes relating to the performative nature of musicking emerge, participants could be additionally empowered to locate and engage with resources in their communities, building relationships and strengthening their base of support as they move forward. The more integrated nature of these relationships and connections to resources could then yield new voices and insights into the work, in reflexive and aspirational ways that could become activist in nature.

Being mindful of the scope of practice may be crucial to clinicians in this process. By nature of research within and uplifting the voices of Black youth from limited-resource communities being as scarce as it is, as well as the population of Black music therapists being relatively low, clinicians may need to do additional work to process through their own unconscious biases about these youth, the communities they come from, and their preferred music. Research suggests this work is worth the effort, with the potential to greatly enhance the participants' quality of life, by providing opportunities for them to be engaged within culturally relevant, responsive, and sustaining ways. These impacts can extend well beyond the limited interaction within the therapeutic relationship, while still feeding and uplifting communities of origin in authentic and profoundly meaningful ways.

Conflicts of interest: None declared.

Natasha Thomas, PhD, is a board-certified music therapist (MT-BC) and Clinical Assistant Professor at Indiana University Purdue University at Indianapolis (IUPUI). She received her undergraduate degree in music therapy from the University of North Dakota (UND), holds a master's degree in special education (also from UND, with an emphasis on visual impairment), and completed her PhD in expressive therapies from Lesley University in Cambridge, MA. Natasha is a committed advocate for creative \& culturally sustaining support for marginalized communities. Her research, clinical, and community-oriented work are all inclusive of emerging technology, as well as the perspectives of disability and queer identities, and the unique ways those perspectives and resources can intersect to impact the quality of life and meaning-making. 


\section{References}

Ansdell, G. (2002). Community music therapy \& the winds of change. Voices, 2(2).

doi:10.15845/voices.v2i2.83

Aramovich, N. P. (2014). The effect of stereotype threat on group versus individual performance. Small Group Research, 45(2), 176-197. doi:10.1177/1046496414523508

Bandura, A. (2005). Adolescent development from an agentic perspective. In T. C. Urdin \& F. Pajares (Eds.), Self-efficacy beliefs of adolescents (pp. 1-45). Charlotte, NC: Information Age Publishing.

Burch, T. (2014). The old Jim Crow: Racial residential segregation and neighborhood imprisonment. LAW \& POLICY, 36(3), 223-255. doi:10.1111/lapo.12022

Chen, G., Gully, S. M., \& Eden, D. (2004). General self-efficacy and self-esteem: Toward theoretical and empirical distinction between correlated selfevaluations. Journal of Organized Behavior, 25, 375-395. doi:10.1002/job.251

Creswell, J. W. (2007). Qualitative inquiry \& research design: Choosing among five approaches. Thousand Oaks, CA: Sage Publications.

Darensbourg, A. M., \& Blake, J. J. (2013). Predictors of achievement in African American students at risk for academic failure: The roles of achievement values and behavioral engagement. Psychology in the Schools, 50(10), 1044-1059. doi:10.1002/pits.21730

Dixon, T. L., Zhang, Y., \& Conrad, K. (2009). Self-esteem, misogyny and afrocentricity: an examination of the relationship between rap music consumption and African American perceptions. Group Processes \& Intergroup Relations, 12(3), 345-360.

Donnetrice, A. (2012). Hip hop, identity, and African American teens. The International Journal of the Humanities, 9(6).

Elpus, K., \& Abril, C. R. (2011). High school music ensemble students in the United States: A demographic profile. Journal of Research in Music Education, 59(2), 128-145. doi:10.1177/0022429411405207

Elsaesser, C., Hong, J. S., \& Voisin, D. R. (2016). Violence exposure and bullying among African American adolescents: Examining the protective role of academic engagements. Children and Youth Services Review, 70, 394-402. doi:10.1016/j.childyouth.2016.10.010

Gaztambide-Fernández, R. A., Saifer, A., \& Desai, C. (2013). "Talent" and the misrecognition of social advantage in specialized arts education. Roeper Review, 35, 124-135.

doi:10.1080/02783193.2013.766964

Harrist, A. W., \& Bradley, D. K. (2002). Social exclusion in the classroom: Teachers and students as agents of change. In J. Aronson (Ed.), Improving academic achievement: Impact of psychological factors on education (pp. 363-383). San Diego, CA: Elsevier Science.

Hense, C. (2015). Forming the youth music action group. Voices, 15(1). doi:10.15845/voices.v1i1.810 
Hoskins, C. N., \& Mariano, C. (2004). Springer Series on the teaching of nursing: Research in nursing and health: Understanding and using quantitative and qualitative methods (2nd ed.). New York, NY: Springer Publishing Company.

lerardi, F., Bottos, M., O’Brian, M. K. (2007). Safe expressions: A community based music therapy program for at risk youth. In V. Camilleri (Ed.), Healing the inner city child: Creative arts therapies with at risk youth (pp. 254-285). London, England: Jessica Kingsley.

Kenny, C. (2004). A holistic framework for aboriginal policy research. Status of Women Canada. Retrieved from http://www.turtleisland.org/resources/hresearch.pdf

Ladson-Billings, G. (2006). From the achievement gap to the education debt: Understanding achievement in U.S. schools [Video]. Available from http://www.nwp.org/cs/public/print/resource/2513.

Lorah, J. A., Sanders, E. A., \& Morrison, S. J. (2014). The relationship between English language learner status and music ensemble participation. Journal of Research in Music Education, 62(3), 23-39.

doi:10.5406/bulcouresmusedu.206.0023

McFerran, K. S., Garrido, S., O'Grady L., Grocke, D., \& Sawyer, S. M. (2015). Examining the relationship between self-reported mood management and music preferences of Australian teenagers. Nordic Journal of Music Therapy, 24(3), 187-203, doi:10.1080/08098131.2014.908942

McTaggart, R. (1997). Participatory action research: International contexts and consequences. New York, NY: State University of New York.

Pyatak, E., \& Muccitelli, L. (2011). Rap music as resistive occupation: constructions of Black identity and culture for performers and their audiences. Journal of Occupational Science, 18(1), 48-61.

Rabaka, R. (2013). The Hip Hop movement: From R\&B and the Civil Rights Movement to rap and the Hip Hop generation. Retrieved from https://ebookcentral-proquest-com.ezproxyles.flo.org

Saunders, J. A. (2010). Identity in music: Adolescents and the music classroom. Action, Criticism, and Theory for Music Education, 9(2), 70-78.

Small, C. (1998). Musicking : The meanings of performing and listening. London: Wesleyan University Press.

Steele, C. M. (2010). Whistling Vivaldi: How stereotypes affect us and what we can do. New York, NY: Norton.

Stige, B. (2002). Culture-centered music therapy. Gilsum, NH: Barcelona Publishers. Stige, B., \& Aarø, L. E. (2012). Invitation to community music therapy. New York: Routledge.

Thomas, N. (2019). Social identity and the music making choices of black/African american youth from limited resource communities (doctoral dissertation). Lesley University, Cambridge, MA. Retrieved from https://digitalcommons.lesley.edu/cgi/viewcontent.cgi?article=1086\&context=expressive_dissertations

Towns, A. R. (2015). The (racial) biases of communication: rethinking media and blackness. Social Identities, 21(5), 474-488. doi:10.1080/13504630.2015.1093469 
Vaillancourt, T., Brittain, H. L., McDougall, P., \& Duku, E. (2013). Longitudinal links between childhood peer victimization, internalizing and externalizing problems, and academic functioning: Developmental cascades. Journal of Abnormal Child Psychology, 41(8), 1203-1215. doi:10.1007/s10802-013-9781-5 